\title{
Report on the First International Workshop on Virtual, Augmented, and Mixed Reality for Human-Robot Interaction
}

\author{
Tom Williams, Daniel Szafir, \\ Tathagata Chakraborti, \\ Heni Ben Amor
}

The first International Workshop on Virtual, Augmented, and Mixed Reality for Human-Robot Interaction (VAMHRI) was held in 2018 in conjunction with the 13th International Conference on Human-Robot Interaction. This inaugural workshop brought together researchers from the fields of humanrobot interaction (HRI), robotics, artificial intelligence, and virtual, augmented, and mixed reality to identify challenges in mixed-reality interactions between humans and robots. The workshop featured a keynote talk from Blair MacIntyre (Mozilla, Georgia Tech), a panel discussion, and 30 papers presented as lightning talks or posters. In this report, we briefly survey the papers presented at the workshop and outline some potential directions for the community.
$\mathrm{T}$ he first International Workshop on Virtual, Augmented, and Mixed Reality for Human-Robot Interaction (VAM-HRI) was held in 2018 in conjunction with the 13th International Conference on Human-Robot Interaction. This workshop brought together researchers from the fields of human-robot interaction (HRI), robotics, artificial intelligence, and virtual, augmented, and mixed reality to identify challenges and opportunities in mixed-reality interactions between humans and robots (Williams et al. 2018). Virtual, augmented, and mixed-reality technologies have been studied for many years in the broader human-computer interaction and computer graphics communities; however, there has been little prior integration with robotics. Recent industry developments are beginning to produce mature, con- 
sumer-grade VR and AR devices, and are thus providing a new opportunity for research that investigates how such technologies might improve human-robot interaction. This was the first workshop of its kind at an academic AI or robotics conference to explore the integration between mixed-reality technologies and autonomous systems. This inaugural workshop featured a keynote talk from Blair MacIntyre (Mozilla, Georgia Tech), a panel discussion, and 30 papers presented as lightning talks or posters. In this report, we provide a brief survey of the papers presented at the workshop.

\section{Virtual Reality for Human-Robot Interaction}

Virtual reality technologies allow their users to be transported to new environments, allowing views into locations that are far away, as well as into locations that exist only virtually. Such technologies are being used in the HRI community to allow immersive remote robot teleoperation and to create new environments in which humans and robots can learn to interact safely and efficiently.

One of the first research areas studied in the field of human-robot interaction was that of teleoperation and telemanipulation, in which humans interact with or control robots with whom they are not spatially or temporally collocated (Fong, Thorpe, and Baur 2001; Goodrich and Schultz 2007). Advances in virtual reality technologies are leading to new work on virtual reality-based teleoperation and telemanipulation interfaces for HRI, exploring how VR teleoperation interfaces might be more immersive and natural than previously investigated systems. At the workshop, eight different VR-based teleoperation interfaces were presented. Rosen et al. ${ }^{1}$ presented the open source ROS reality package for VR-based robot teleoperation. Allspaw et al. investigated different types of visualizations useful for robot teleoperation. Gaurav et al. and Tran et al. presented hands-free VR teleoperation interfaces, and Bennett et al. presented a suit-based exo-suit teleoperation interface. Oh et al., Lager, Topp, and Malec, and Haring et al. presented VR-based teleoperation interfaces designed for virtual tours, unmanned surface vehicles, and swarms of aerial robots, respectively.

Researchers are also using VR to explore human interactions with simulated robots in entirely virtual environments. The majority of these projects focus on using immersive virtual reality simulations to allow humans to efficiently train robots. Whitney, Rosen, and Tellex and Tan et al. discussed the use of virtual reality for collecting human demonstrations for learning from demonstration, and Iuzzolino, Walker, and Szafir and Stramandinoli et al. demonstrated the ability of demonstrations captured in this way to generalize to real-world contexts. Taking a slightly different approach, Hansen et al. used virtual reality to evaluate human perceptions of pre- trained robot behaviors. Finally, while the previously discussed approaches were used for training and evaluating robot behaviors, Goedicke et al. used virtual reality to train human drivers, and Novitzky et al. used virtual reality to jointly train teams of humans and robots.

\section{Augmented and Mixed Reality for Human-Robot Interaction}

Unlike virtual reality technologies, augmented and mixed-reality systems do not attempt to transport their users to new environments, but instead enrich user experiences by overlaying virtual imagery on top of the real environment.

Researchers at the International Workshop on Virtual, Augmented, and Mixed Reality for HumanRobot Interaction presented approaches that used augmented and mixed-reality technologies to create novel interfaces for interacting with robots across all stages of a robot's lifecycle, from design (Peters et. al) to calibration (Schönheits and Krebs) to user training (Sportillo et. al), and across a number of different applications, including manufacturing (Bagchi and Marvel) and assistive technologies (Arboleda, Pascher, and Gerken).

In addition, a number of approaches were presented in which these technologies were used to provide robots with new ways to implicitly or explicitly communicate their intentions to human teammates, investigating how to communicate drivers' intentions (Chakraborti, Dudley, and Kambhampati), robots' trajectories (Zu Borgsen et al.; Chakraborti et al.; Cheli et al.), intended actions (Chakraborti et al.; Cheli et al.), abrupt changes in direction (Katzakis and Steinicke), desires with respect to human actions (Amor et al.) or states of the world (Chakraborti et al.), sensor data (Cheli et al.), and intended referents during communication (Amor et al.; Williams). Finally, Quintero et al. presented an interface for communicating and dynamically modifying the trajectories of robot manipulators, while Chakraborti et al. allowed for dynamic replanning at the task level during execution.

\section{Invited Talk}

The invited talk was given by Blair MacIntyre, a principal research scientist in the Emerging Technologies group at Mozilla and a professor of interactive computing at Georgia Tech. His talk examined a number of research areas in which he saw the most benefit of augmented and mixed reality for human-robot interaction, including visualizing information regarding robots' perceptions, states, and plans. His talk concluded with a live demo of Mozilla's WebXR framework and a discussion of its potential benefits for HRI. 


\section{Conclusion and Future Directions}

The inaugural International Workshop on Virtual, Augmented, and Mixed Reality for Human-Robot Interaction at HRI 2018 was successful along a number of criteria. First, it brought together researchers from across academe, industry, and government, and from across the Americas, Europe, and Asia. Second, it clarified a set of four research themes of interest to this community: teleoperation through virtual reality, interaction training in virtual reality simulations, augmented interfaces for human-robot interaction, and intention communication through augmented and mixed reality. And finally, it made clear to the greater HRI community not only the amount of interest in this nascent subfield, but also the broad space of possible research problems that the emerging technologies in virtual, augmented, and mixed reality can help the field of HRI address.

The workshop-organizing committee plans to take the following steps to continue the momentum captured in this inaugural symposium: (1) we are planning a special issue on virtual, augmented, and mixed reality for human-robot interaction, in a journal to be determined, (2) we are writing a survey paper to tie what was discussed at this workshop into the richer research literature of papers that have recently been presented in this area, and (3) we are planning to organize a follow-up workshop next year.

The workshop was organized by Tom Williams, Daniel Szafir, Tathagata Chakraborti, and Heni BenAmor. The workshop featured 30 papers, with contributions from all over the world (Asia and Oceania, 4.1 percent; Europe, 33.6 percent; North America, 62.3 percent) and across different disciplines (government, 3.3 percent; industry, 11.5 percent; academe, 85.2 percent) The workshop was heavily attended, with more than 75 participants. The papers presented at the workshop are available at vamhri.xyz.

\section{Notes}

1. Teams are cited here as they appear on the accompanying workshop papers, available at vam-hri.xyz.

\section{References}

Fong, T.; Thorpe, C.; and Baur, C. 2001. Collaboration, Dialogue, and Human-Robot Interaction. In Robotics Research: The 10th International Symposium, edited by R. A. Jarvis and A. Zelinsky. Berlin: Springer.

Goodrich, M. A., and Schultz, A. C. 2007. Human-Robot Interaction: A Survey. Foundations and Trends in HumanComputer Interaction 1(3): 203-75.

Williams, T.; Szafir, D.; Chakraborti, T.; and Ben Amor, H. 2018. Virtual, Augmented, and Mixed Reality for HumanRobot Interaction. In Companion of the 2018 ACM/IEEE International Conference on Human-Robot Interaction, 403-4. New York: Association for Computing Machinery.
Tom Williams is an assistant professor of computer science at Colorado School of Mines, where he directs the Mines Interactive Robotics Research (MIRROR) Lab. His research focuses on enabling and understanding natural languagebased human-robot interaction, especially as applied to assistive and search-and-rescue robotics. Williams previously served as program committee cochair for the 2016 HRI Pioneers workshop and the AAAI Fall Symposium on AI for HRI, special track session chair for EAAI, and senior program committee member for AAAI.

Daniel Szafir is an assistant professor in the Department of Computer Science and ATLAS Institute at the University of Colorado Boulder, where he directs the Interactive Robotics and Novel Technologies (IRON) Lab. His research at the intersection of robotics and human-computer interaction (HCI) focuses on investigating how novel technologies can mediate interactions between people and autonomous systems. Szafir has previously served as the videos and demos cochair for HRI 2017, an organizing committee member for the RSS 2017 Workshop on Bridging the Gap in Space Robotics, the panel chair for the 2015 HRI Pioneers workshop, and a program committee member for HRI, RO-MAN, CHI, RSS, and ARSO.

Tathagata Chakraborti is a senior PhD student at Arizona State University working in the Yochan Lab with Dr. Subbarao Kambhampati. His research interests include planning with humans in the loop, applications in task planning for human-robot teaming and cohabitation, and proactive decision support. He has been on the organizing team for the Workshop on Multiagent Interaction without Prior Coordination (MIPC) at AAMAS-17 and Workshop on Explainable AI (XAI) at IJCAI 2017, as well as on the Review Process Committee of IJCAI 2016. Recently, he was the team lead of AERobotics Robotics, which featured in the US Finals of the Microsoft Imagine Cup 2017.

Heni Ben Amor is an assistant professor at Arizona State University, where he heads the ASU Interactive Robotics Lab. Prior to that, he was a research scientist at the Institute for Robotics and Intelligent Machines at Georgia Tech. He studied computer science at the University of Koblenz-Landau (GER) and earned a PhD in robotics from the Technical University Freiberg and the University of Osaka in 2010, and was a postdoctoral scholar at the Technical University Darmstadt. His research topics focus on artificial intelligence, machine learning, human-robot interaction, robot vision, and automatic motor skill acquisition. He received the Daimler and Benz Fellowship, as well as several best paper awards at major robotics and AI conferences. He is a program committee member for various AI and robotics conferences including AAAI, IJCAI, IROS, and ICRA. 